\title{
BÄCKLUND TRANSFORMATIONS FOR NON-COMMUTATIVE ANTI-SELF-DUAL YANG-MILLS EQUATIONS
}

\author{
CLAIRE R. GILSON \\ Department of Mathematics, University of Glasgow, Glasgow G12 8QW, UK \\ e-mail:crg@maths.gla.ac.uk \\ MASASHI HAMANAKA \\ Department of Mathematics, University of Nagoya, Nagoya 464-8602, Japan \\ e-mail: hamanaka@math.nagoya-u.ac.jp \\ and JONATHAN J. C. NIMMO \\ Department of Mathematics, University of Glasgow, Glasgow G12 8QW, UK \\ e-mail: j.nimmo@maths.gla.ac.uk
}

\begin{abstract}
We present Bäcklund transformations for the non-commutative antiself-dual Yang-Mills equations where the gauge group is $G=G L(2)$ and use it to generate a series of exact solutions from a simple seed solution. The solutions generated by this approach are represented in terms of quasi-determinants and belong to a noncommutative version of the Atiyah-Ward ansatz. In the commutative limit, our results coincide with those by Corrigan, Fairlie, Yates and Goddard.
\end{abstract}

2000 Mathematics Subject Classification. 35Q58, 46L55, 70S15.

1. Introduction. Non-commutative (NC) extensions of integrable systems and soliton theory have been studied intensively for the last few years in various contexts of both mathematics and physics (for reviews, see, e.g. [22]). In particular, the extension to $\mathrm{NC}$ spaces has drawn much attention in physics, because in gauge theories this kind of $\mathrm{NC}$ extension corresponds to the presence of background magnetic fields, and various applications have been made successfully (for reviews, see, e.g. [21]).

In commutative gauge theories, the anti-self-dual Yang-Mills (ASDYM) equation is quite important. The finite-action solutions, that is the instanton solutions, play key roles in field theories and in four-dimensional geometry. In integrable systems, it is also very important since many lower-dimensional integrable equations such as the Korteweg-de Vries (KdV) equation and the Toda field equation can be derived as reductions of this ASDYM equation. This connection was first remarked on by Richard Ward [33] (known as Ward's conjecture) and is summarized elegantly in the book of Mason and Woodhouse [26] using twistor theory.

Following the work of Nekrasov and Schwarz [27], the NC ASDYM equation has been investigated from various viewpoints. It is actually integrable in some sense [2, 3, 17-20, 23, 24, 32] and contain new solutions special to NC spaces. Furthermore, many NC integrable equations are proved to be reductions of NC ASDYM equation [15], and therefore it is now worth studying the integrable aspects of the NC ASDYM equation in detail and applying the results to lower-dimensional integrable equations. 
In the present paper, we give Bäcklund transformations for NC ASDYM equation where the gauge group is $G=G L(2)$ and use it to generate a series of exact solutions from a simple seed solution. The solutions generated by this approach are represented in terms of quasi-determinants [9] and belong to a NC version of the Atiyah-Ward ansatz [1]. Quasi-determinants appear also in the construction of exact soliton solutions in lower-dimensional integrable equations such as $\mathrm{KdV}$ equation $[\mathbf{6}, \mathbf{7}, \mathbf{1 1 - 1 4}, \mathbf{1 6}, \mathbf{2 5}, \mathbf{2 8}$ ], where they play the role that determinants do in the corresponding commutative integrable systems. In the commutative limit, our results coincide with those by Corrigan et al. [5].

We note that in our treatment, all multiplication is associative but is not assumed to be commutative. Hence, the results we obtain are available not only for discussion on NC flat spaces which are realized by replacement of all products with star-products, but also in other NC settings such as matrix or quarternion-valued ASDYM equations.

2. Brief review of quasi-determinants. In this section, we give a brief introduction to quasi-determinants, introduced by Gelfand and Retakh [9], in which a few of the key properties which play important roles in the following sections are described. More detailed discussion is seen in the survey [8].

Quasi-determinants are defined in terms of inverse matrices and we suppose the existence of all matrix inverses referred to. Let $A=\left(a_{i j}\right)$ be a $n \times n$ matrix and $B=\left(b_{i j}\right)$ be the inverse matrix of $A$, that is, $A B=B A=1$. Here the matrix entries belong to a noncommutative ring. Quasi-determinants of $A$ are defined formally as the inverses of the entries in $B:|A|_{i j}:=b_{j i}^{-1}$. In the case that variables commute, this is reduced to

$$
|A|_{\ddot{j}}=(-1)^{i+j} \frac{\operatorname{det} A}{\operatorname{det} \tilde{A}^{\ddot{j}}},
$$

where $\tilde{A}^{\ddot{j}}$ is the matrix obtained from $A$ by deleting the $i$ th row and the $j$ th column.

We can also write down a more explicit definition of quasi-determinants. In order to see this, let us recall the following formula for the inverse of a square $2 \times 2$ block square matrix:

$$
\left[\begin{array}{ll}
A & B \\
C & d
\end{array}\right]^{-1}=\left[\begin{array}{cc}
A^{-1}+A^{-1} B S^{-1} C A^{-1} & -A^{-1} B S^{-1} \\
-S^{-1} C A^{-1} & S^{-1}
\end{array}\right]
$$

where $A$ is a square matrix, $d$ is a single element and $B$ and $C$ are column and row vectors of appropriate length and $S=d-C A^{-1} B$ is called a Schur complement. In fact this formula is valid for $A, B, C$ and $d$ in any ring not just for matrices. Thus, the quasideterminant associated with the bottom right element is simply $S$. By choosing an appropriate partitioning, any entry in the inverse of a square matrix can be expressed as the inverse of a Schur complement and hence quasi-determinants can also be defined recursively by

$$
|A|_{i j}=a_{i j}-\sum_{i^{\prime}(\neq i), j^{\prime}(\neq j)} a_{i i^{\prime}}\left(\left(\tilde{A}^{\ddot{j}}\right)^{-1}\right)_{i^{\prime} j^{\prime}} a_{j^{\prime} j}=a_{\ddot{j}}-\sum_{i^{\prime}(\neq i), j^{\prime}(\neq j)} a_{i i^{\prime}}\left(\left|\tilde{A}^{\ddot{j}}\right|_{j^{\prime} i^{\prime}}\right)^{-1} a_{j^{\prime} j}
$$


It is sometimes convenient to use the following alternative notation in which a box is drawn about the corresponding entry in the matrix:

$$
|A|_{i j}=\left|\begin{array}{ccccc}
a_{11} & \cdots & a_{1 j} & \cdots & a_{1 n} \\
\vdots & & \vdots & & \vdots \\
a_{i 1} & & a_{i j} & & a_{i n} \\
\vdots & & \vdots & & \vdots \\
a_{n 1} & \cdots & a_{n j} & \cdots & a_{n n}
\end{array}\right| .
$$

Quasi-determinants have various interesting properties similar to those of determinants. Among them, the following ones play important roles in this paper. In the block matrices given in these results, lowercase letters denote single entries and uppercase letters denote matrices of compatible dimensions so that the overall matrix is square.

- NC Jacobi identity [11]

A simple and useful special case of the NC Sylvester's Theorem [9] is

$$
\left|\begin{array}{ccc}
A & B & C \\
D & f & g \\
E & h & i
\end{array}\right|=\left|\begin{array}{cc}
A & C \\
E & i
\end{array}\right|-\left|\begin{array}{cc}
A & B \\
E & h
\end{array}\right|\left|\begin{array}{cc}
A & B \\
D & f
\end{array}\right|^{-1}\left|\begin{array}{cc}
A & C \\
D & g
\end{array}\right| .
$$

- Homological relations [9]

$$
\left|\begin{array}{lll}
A & B & C \\
D & f & g \\
E & h & i
\end{array}\right|=\left|\begin{array}{lll}
A & B & C \\
D & f & g \\
E & h & i
\end{array}\right|\left|\begin{array}{lll}
A & B & C \\
D & f & g \\
0 & 0 & 1
\end{array}\right|,\left|\begin{array}{ccc}
A & B & C \\
D & f & g \\
E & h & i
\end{array}\right|=\left|\begin{array}{ccc}
A & B & 0 \\
D & f & 0 \\
E & h & 1
\end{array}\right|\left|\begin{array}{lll}
A & B & C \\
D & f & g \\
E & h & i
\end{array}\right| .
$$

- A derivative formula for quasi-determinants [11]

$$
\left|\begin{array}{cc}
A & B \\
C & d
\end{array}\right|^{\prime}=\left|\begin{array}{cc}
A & B^{\prime} \\
C & d^{\prime}
\end{array}\right|+\sum_{k=1}^{n}\left|\begin{array}{cc}
A & \left(A_{k}\right)^{\prime} \\
C & \left(C_{k}\right)^{\prime}
\end{array}\right|\left|\begin{array}{cc}
A & B \\
e_{k}^{t} & 0
\end{array}\right|,
$$

where $A_{k}$ is the $k$ th column of a matrix $A$ and $e_{k}$ is the column $n$-vector $\left(\delta_{i k}\right)$ (i.e. 1 in the $k$ th row and 0 elsewhere).

3. Bäcklund transformation for the NC ASDYM equation. In this section, we give Bäcklund transformations which leaves invariant the NC ASDYM equation, or equivalently, the NC Yang's equation for $G=G L(2)$. We also present explicit expressions, in terms of quasi-determinants, for the solutions generated in this way. This transformation is a NC version of the so-called Corrigan-Fairlie-Yates-Goddard ( CFYG) transformation [5]. It generates a series of exact solutions which belong to $\mathrm{NC}$ version of the Atiyah-Ward ansatz [1] labelled by a positive integer.

3.1. The NC ASDYM equation and the NC Yang's equation. Let us consider a special representation of the NC ASDYM equation for $G=G L(2)$,

$$
\partial_{z}\left(J^{-1} \partial_{\tilde{z}} J\right)-\partial_{w}\left(J^{-1} \partial_{\tilde{w}} J\right)=0,
$$


where $z, \tilde{z}, w, \tilde{w}$ denote double null coordinates of four-dimensional space [26]. Equation (3.1) is called the $N C$ Yang's equation and the gauge-invariant matrix $J$ is called Yang's $J$-matrix. Gauge fields are obtained from a solution $J$ of the NC Yang's equation via a decomposition $J=\tilde{h}^{-1} h$ :

$$
A_{z}=-\left(\partial_{z} h\right) h^{-1}, \quad A_{w}=-\left(\partial_{w} h\right) h^{-1}, \quad A_{\tilde{z}}=-\left(\partial_{\tilde{z}} \tilde{h}\right) \tilde{h}^{-1}, \quad A_{\tilde{w}}=-\left(\partial_{\tilde{w}} \tilde{h}\right) \tilde{h}^{-1} .
$$

In order to discuss Bäcklund transformations for the NC Yang's equation, we parameterize the $2 \times 2$ matrix $J$ as

$$
J=\left[\begin{array}{cc}
f-g b^{-1} e & -g b^{-1} \\
b^{-1} e & b^{-1}
\end{array}\right] .
$$

This parameterization is always possible when $f$ and $b$ are non-singular. In comparison with the commutative case, where only $f$ appears, in the NC setting we need to introduce another valuable $b$. In the commutative limit we may choose $b=f$.

Then the NC Yang's equation (3.1) is decomposed as

$$
\begin{array}{r}
\partial_{z}\left(f^{-1} g_{\tilde{z}} b^{-1}\right)-\partial_{w}\left(f^{-1} g_{\tilde{w}} b^{-1}\right)=0, \quad \partial_{\tilde{z}}\left(b^{-1} e_{z} f^{-1}\right)-\partial_{\tilde{w}}\left(b^{-1} e_{w} f^{-1}\right)=0, \\
\partial_{z}\left(b_{\tilde{z}} b^{-1}\right)-\partial_{w}\left(b_{\tilde{w}} b^{-1}\right)-e_{z} f^{-1} g_{\tilde{z}} b^{-1}+e_{w} f^{-1} g_{\tilde{w}} b^{-1}=0, \\
\partial_{z}\left(f^{-1} f_{\tilde{z}}\right)-\partial_{w}\left(f^{-1} f_{\tilde{w}}\right)-f^{-1} g_{\tilde{z}} b^{-1} e_{z}+f^{-1} g_{\tilde{w}} b^{-1} e_{w}=0,
\end{array}
$$

where subscripts denote partial derivatives.

A gauge fixing corresponds to a decomposition of $J$ into matrices $h$ and $\tilde{h}$. There is a simple and useful decomposition corresponding to the above parameterization of $J$ :

$J=\tilde{h}^{-1} h=\left[\begin{array}{ll}1 & g \\ 0 & b\end{array}\right]^{-1}\left[\begin{array}{ll}f & 0 \\ e & 1\end{array}\right], \quad J^{-1}=\left[\begin{array}{ll}f & 0 \\ e & 1\end{array}\right]^{-1}\left[\begin{array}{ll}1 & g \\ 0 & b\end{array}\right]=\left[\begin{array}{cc}f^{-1} & f^{-1} g \\ -e f^{-1} & b-e f^{-1} g\end{array}\right]$.

By using these formulae, we can find the gauge fields and the field strength in terms of $b, e, f$ and $g$.

3.2. NC CFYG transformation. Now we describe the NC CFYG transformation explicitly. It is a composition of the following two Bäcklund transformations for the decomposed NC Yang's equations (3.3):

- $\beta$-transformation [26]:

$$
\begin{aligned}
& e_{w}^{\text {new }}=f^{-1} g_{\tilde{z}} b^{-1}, \quad e_{z}^{\text {new }}=f^{-1} g_{\tilde{w}} b^{-1}, \quad g_{\tilde{z}}^{\text {new }}=b^{-1} e_{w} f^{-1}, \quad g_{\tilde{w}}^{\text {new }}=b^{-1} e_{z} f^{-1}, \\
& f^{\text {new }}=b^{-1}, \quad b^{\text {new }}=f^{-1} .
\end{aligned}
$$

The first four equations can be interpreted as integrability conditions for the first two equations in (3.3). We can easily check that the last two equations in (3.3) are invariant under this transformation. Also, it is clear that $\beta \circ \beta$ is the identity transformation.

- $\gamma_{0}$-transformation:

$$
\left[\begin{array}{ll}
f^{\text {new }} & g^{\text {new }} \\
e^{\text {new }} & b^{\text {new }}
\end{array}\right]=\left[\begin{array}{ll}
b & e \\
g & f
\end{array}\right]^{-1}=\left[\begin{array}{ll}
\left(b-e f^{-1} g\right)^{-1} & \left(g-f e^{-1} b\right)^{-1} \\
\left(e-b g^{-1} f\right)^{-1} & \left(f-g b^{-1} e\right)^{-1}
\end{array}\right]
$$


This follows from the fact that the transformation $\gamma_{0}: J \mapsto J^{\text {new }}$ is equivalent to the simple conjugatation $J^{\text {new }}=C^{-1} J C, C=\left[\begin{array}{ll}0 & 1 \\ 1 & 0\end{array}\right]$, which clearly leaves the NC Yang's equation (3.1) invariant. Relation (3.5) is derived by comparing elements in this transformation. It is a trivial fact that $\gamma_{0} \circ \gamma_{0}$ is the identity transformation.

3.3. Exact NC Atiyah-Ward ansatz solutions. Now we construct exact solutions by using a chain of Bäcklund transformations from a seed solution. Let us consider $b=e=f=g=\Delta_{0}^{-1}$, then we can easily find that the decomposed NC Yang's equation is reduced to a NC linear equation $\left(\partial_{z} \partial_{\tilde{z}}-\partial_{w} \partial_{\tilde{w}}\right) \Delta_{0}=0$. (We note that for the Euclidean space, this is the NC Laplace equation because of the reality condition $\bar{w}=-\tilde{w}$.) Hence we can generate two series of exact solutions $R_{m}$ and $R_{m}^{\prime}$ by iterating the $\beta$ - and $\gamma_{0^{-}}$ transformations one after the other as follows (the seed solution $b=e=f=g=\Delta_{0}^{-1}$ belongs to $R_{1}$ ):

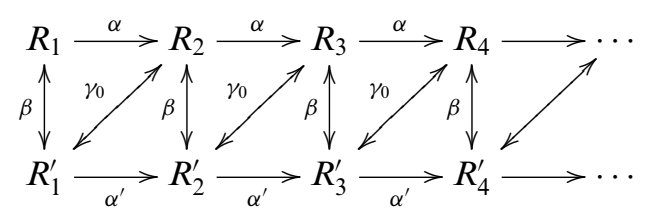

where $\alpha=\gamma_{0} \circ \beta: R_{m} \rightarrow R_{m+1}$ and $\alpha^{\prime}=\beta \circ \gamma_{0}: R_{m}^{\prime} \rightarrow R_{m+1}^{\prime}$. These two kinds of series of solutions in fact arise from some class of NC Atiyah-Ward ansatz. ${ }^{1}$ The explicit form of the solutions $R_{m}$ or $R_{m}^{\prime}$ can be represented in terms of quasideterminants whose elements $\Delta_{r}(r=-m+1,-m+2, \ldots, m-1)$ satisfy

$$
\frac{\partial \Delta_{r}}{\partial z}=-\frac{\partial \Delta_{r+1}}{\partial \tilde{w}}, \quad \frac{\partial \Delta_{r}}{\partial w}=-\frac{\partial \Delta_{r+1}}{\partial \tilde{z}}, \quad-m+1 \leq r \leq m-2 \quad(m \geq 2),
$$

which implies that every element $\Delta_{r}$ is a solution of the NC linear equation $\left(\partial_{z} \partial_{\tilde{z}}-\right.$ $\left.\partial_{w} \partial_{\tilde{w}}\right) \Delta_{r}=0$. The results are as follows:

- NC Atiyah-Ward ansatz solutions $R_{m}$

NC Atiyah-Ward ansatz solutions $R_{m}$ are represented by the explicit form of elements $b_{m}, e_{m}, f_{m}, g_{m}$ in $J_{m}$ as follows:

$$
\begin{aligned}
b_{m} & =\left|\begin{array}{cccc}
\Delta_{0} & \Delta_{-1} & \cdots & \Delta_{1-m} \\
\Delta_{1} & \Delta_{0} & \cdots & \Delta_{2-m} \\
\vdots & \vdots & \ddots & \vdots \\
\Delta_{m-1} & \Delta_{m-2} & \cdots & \Delta_{0}
\end{array}\right|^{-1}, f_{m}=\left|\begin{array}{cccc}
\Delta_{0} & \Delta_{-1} & \cdots & \Delta_{1-m} \\
\Delta_{1} & \Delta_{0} & \cdots & \Delta_{2-m} \\
\vdots & \vdots & \ddots & \vdots \\
\Delta_{m-1} & \Delta_{m-2} & \cdots & \Delta_{0}
\end{array}\right|^{-1}, \\
e_{m} & =\left|\begin{array}{cccc}
\Delta_{0} & \Delta_{-1} & \cdots & \Delta_{1-m} \\
\Delta_{1} & \Delta_{0} & \cdots & \Delta_{2-m} \\
\vdots & \vdots & \ddots & \vdots \\
\Delta_{m-1} & \Delta_{m-2} & \cdots & \Delta_{0}
\end{array}\right|, g_{m}=\left|\begin{array}{cccc}
\Delta_{0} & \Delta_{-1} & \cdots & \Delta_{1-m} \\
\Delta_{1} & \Delta_{0} & \cdots & \Delta_{2-m} \\
\vdots & \vdots & \ddots & \vdots \\
\Delta_{m-1} & \Delta_{m-2} & \cdots & \Delta_{0}
\end{array}\right|^{-1}
\end{aligned}
$$

In the commutative limit, we can easily see that $b_{m}=f_{m}$. The ansatz $R_{1}$ leads to so-called Corrigan-Fairlie-'t Hooft-Wilczek (CFtHW) ansatz [4].

$\overline{{ }^{1}}$ This is discussed in our forthcoming paper in detail [10]. 
- NC Atiyah-Ward ansatz solutions $R_{m}^{\prime}$

NC Atiyah-Ward ansatz solutions $R_{m}^{\prime}$ are represented by the explicit form of elements $b_{m}^{\prime}, e_{m}^{\prime}, f_{m}^{\prime}, g_{m}^{\prime}$ in $\tilde{J}_{m}$ as follows:

$$
\begin{aligned}
b_{m}^{\prime} & =\left|\begin{array}{cccc}
\mid \Delta_{0} & \Delta_{-1} & \cdots & \Delta_{1-m} \\
\Delta_{1} & \Delta_{0} & \cdots & \Delta_{2-m} \\
\vdots & \vdots & \ddots & \vdots \\
\Delta_{m-1} & \Delta_{m-2} & \cdots & \Delta_{0}
\end{array}\right|, \quad f_{m}^{\prime}=\left|\begin{array}{cccc}
\Delta_{0} & \Delta_{-1} & \cdots & \Delta_{1-m} \\
\Delta_{1} & \Delta_{0} & \cdots & \Delta_{2-m} \\
\vdots & \vdots & \ddots & \vdots \\
\Delta_{m-1} & \Delta_{m-2} & \cdots & \Delta_{0}
\end{array}\right|, \\
e_{m}^{\prime} & =\left|\begin{array}{cccc}
\Delta_{-1} & \Delta_{-2} & \cdots & \Delta_{-m} \\
\Delta_{0} & \Delta_{-1} & \cdots & \Delta_{1-m} \\
\vdots & \vdots & \ddots & \vdots \\
\Delta_{m-2} & \Delta_{m-3} & \cdots & \Delta_{-1}
\end{array}\right|, \quad g_{m}^{\prime}=\left|\begin{array}{cccc}
\Delta_{1} & \Delta_{0} & \cdots & \Delta_{2-m} \\
\Delta_{2} & \Delta_{1} & \cdots & \Delta_{3-m} \\
\vdots & \vdots & \ddots & \vdots \\
\Delta_{m} & \Delta_{m-1} & \cdots & \Delta_{1}
\end{array}\right| .
\end{aligned}
$$

In the commutative case, $b_{m}^{\prime}=f_{m}^{\prime}$ also holds. For $m=1$, we get $b_{1}^{\prime}=f_{1}^{\prime}=\Delta_{0}, e_{1}^{\prime}=$ $\Delta_{-1}, g_{1}^{\prime}=\Delta_{1}$ and then the relation (3.6) implies that $e_{1, z}^{\prime}=f_{1, \tilde{w}}^{\prime}, e_{1, w}^{\prime}=f_{1, \tilde{z}}^{\prime}, f_{1, z}^{\prime}=$ $g_{1, \tilde{w}}^{\prime}, f_{1, w}^{\prime}=g_{1, \tilde{z}}^{\prime}$, and leads to the CFtHW ansatz which was first pointed out by Yang [34].

We can also present a compact form of the whole Yang's matrix $J$ in terms of a single quasi-determinants expanded by a $2 \times 2$ submatrix:

$$
J_{m}^{\prime}=\left[\begin{array}{cc}
f_{m}^{\prime}-g_{m}^{\prime} b_{m}^{\prime-1} e_{m}^{\prime} & -g_{m}^{\prime} b_{m}^{\prime-1} \\
b_{m}^{\prime-1} e_{m}^{\prime} & b_{m}^{\prime-1}
\end{array}\right]=\left|\begin{array}{cccccc}
\Delta_{0} & \Delta_{-1} & \cdots & \Delta_{1-m} & \Delta_{-m} & -1 \\
\Delta_{1} & \Delta_{0} & \cdots & \Delta_{2-m} & \Delta_{1-m} & 0 \\
\vdots & \vdots & \ddots & \vdots & \vdots & \vdots \\
\Delta_{m-1} & \Delta_{m-2} & \cdots & \Delta_{0} & \Delta_{-1} & 0 \\
\Delta_{m} & \Delta_{m-1} & \cdots & \Delta_{1} & \Delta_{0} & 0 \\
1 & 0 & \cdots & 0 & 0 & 0
\end{array}\right|
$$

and

$$
J_{m}^{\prime-1}=\left[\begin{array}{cc}
f_{m}^{\prime-1} & f_{m}^{\prime-1} g_{m}^{\prime} \\
-e_{m}^{\prime} f_{m}^{\prime-1} & b_{m}^{\prime}-e_{m}^{\prime} f_{m}^{\prime-1} g_{m}^{\prime}
\end{array}\right]=\left|\begin{array}{cccccc}
0 & 0 & 0 & \cdots & 0 & 1 \\
0 & \Delta_{0} & \Delta_{-1} & \cdots & \Delta_{1-m} & \Delta_{-m} \\
0 & \Delta_{1} & \Delta_{0} & \cdots & \Delta_{2-m} & \Delta_{1-m} \\
\vdots & \vdots & \vdots & \ddots & \vdots & \vdots \\
0 & \Delta_{m-1} & \Delta_{m-2} & \cdots & \Delta_{0} & \Delta_{-1} \\
-1 & \Delta_{m} & \Delta_{m-1} & \cdots & \Delta_{1} & \Delta_{0}
\end{array}\right|
$$

Because $J$ is gauge invariant, this shows that the present Bäcklund transformation is not just a gauge transformation but a non-trivial one. The proof of these representations is given in Appendix A. 
The $\gamma_{0}$-transformation is proved simply using NC Jacobi identity (2.4) applied to the four corner elements. ${ }^{2}$ For example,

$$
\begin{aligned}
& b_{m+1}^{-1}=\left|\begin{array}{ccc}
\Delta_{0} & \cdots & \Delta_{1-m} \\
\vdots & \ddots & \vdots \\
\Delta_{m-1} & \cdots & \Delta_{0}
\end{array}\right|-\left.\left|\begin{array}{ccc}
\Delta_{1} & \cdots & \Delta_{2-m} \\
\vdots & \ddots & \vdots \\
\Delta_{m} & \cdots & \Delta_{1}
\end{array}\right| \begin{array}{|ccc}
\Delta_{0} & \cdots & \Delta_{1-m} \\
\vdots & \ddots & \vdots \\
\Delta_{m-1} & \cdots & \Delta_{0}
\end{array}\right|^{-1}\left|\begin{array}{ccc}
\Delta_{-1} & \cdots & \Delta_{-m} \\
\vdots & \ddots & \vdots \\
\Delta_{m-2} & \cdots & \Delta_{-1}
\end{array}\right| \\
& =f_{m}^{\prime}-g_{m}^{\prime} b_{m}^{-1} e_{m}^{\prime} \text {. }
\end{aligned}
$$

The proof of the $\beta$-transformation uses both the NC Jacobi identity and also the homological relations (2.5). We will prove the first equation in the $\beta$-transformation, that is, if the $\Delta_{i}$ satisfy $\Delta_{i, w}=-\Delta_{i+1, z}$ then

$$
e_{m, w}^{\prime}=f_{m}^{-1} g_{m, \tilde{z}} b_{m}^{-1} .
$$

The right-hand side is equal to

$$
-b_{m}^{\prime} g_{m}\left(g_{m}^{-1}\right) \tilde{z} g_{m} f_{m}^{\prime} .
$$

In this, it follows from (2.5) that the first two and last two factors are

$$
b_{m}^{\prime} g_{m}=\left|\begin{array}{cccc}
0 & \Delta_{-1} & \cdots & \Delta_{1-m} \\
0 & \Delta_{0} & \cdots & \Delta_{2-m} \\
\vdots & \vdots & & \vdots \\
0 & \Delta_{m-3} & \cdots & \Delta_{-1} \\
1 & \Delta_{m-2} & \cdots & \Delta_{0}
\end{array}\right|, \quad g_{m} f_{m}^{\prime}=\left|\begin{array}{ccccc}
\Delta_{0} & \Delta_{-1} & \cdots & \Delta_{2-m} & \Delta_{1-m} \\
\vdots & \vdots & & \vdots & \vdots \\
\Delta_{m-2} & \Delta_{m-3} & \cdots & \Delta_{0} & \Delta_{-1} \\
1 & 0 & \cdots & 0 & 0
\end{array}\right|
$$

Next, from (2.6), we have

$$
\begin{gathered}
\left(g_{m}^{-1}\right)_{\tilde{z}}=\left|\begin{array}{cccc}
\Delta_{0, \tilde{z}} & \Delta_{-1} & \cdots & \Delta_{1-m} \\
\Delta_{1, \tilde{z}} & \Delta_{0} & \cdots & \Delta_{2-m} \\
\vdots & \vdots & & \vdots \\
\Delta_{m-2, \tilde{z}} & \Delta_{m-3} & \cdots & \Delta_{-1} \\
\Delta_{m-1, \tilde{z}} & \Delta_{m-2} & \cdots & \Delta_{0}
\end{array}\right| \\
+\sum_{k=1}^{m-1}\left|\begin{array}{cccc}
\Delta_{-k, \tilde{z}} & \Delta_{-1} & \cdots & \Delta_{1-m} \\
\Delta_{1-k, \tilde{z}} & \Delta_{0} & \cdots & \Delta_{2-m} \\
\vdots & \vdots & & \vdots \\
\Delta_{m-2-k, \tilde{z}} & \Delta_{m-3} & \cdots & \Delta_{-1} \\
\Delta_{m-1-k, \tilde{z}} & \Delta_{m-2} & \cdots & \Delta_{0}
\end{array}\right| \begin{array}{cccccc}
\Delta_{0} & \Delta_{-1} & \cdots & \Delta_{-k} & \cdots & \Delta_{1-m} \\
\vdots & \vdots & & \vdots & & \\
\Delta_{m-2} & \Delta_{m-3} & \cdots & \Delta_{m-2-k} & \cdots & \Delta_{-1} \\
0 & 0 & \cdots & 1 & \cdots & 0
\end{array} \mid .
\end{gathered}
$$

The effect of the left and right factors on this expression is to move expansion points as specified in (2.5) and so, after moving the expansion column (row) in the first (second)

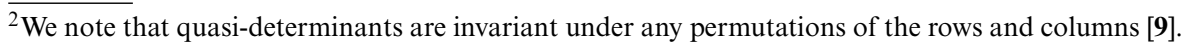


factors to the rightmost (top), we get

$$
\begin{aligned}
& f_{m}^{-1} g_{m, \tilde{z}} b_{m}^{-1}=-\left|\begin{array}{cccc}
\Delta_{-1} & \cdots & \Delta_{1-m} & \Delta_{1-m, \tilde{z}} \\
\Delta_{0} & \cdots & \Delta_{2-m} & \Delta_{2-m, \tilde{z}} \\
\vdots & & \vdots & \vdots \\
\Delta_{m-3} & \cdots & \Delta_{-1} & \Delta_{-1, \tilde{z}} \\
\Delta_{m-2} & \cdots & \Delta_{0} & \Delta_{0, \tilde{z}}
\end{array}\right| \\
& -\sum_{k=0}^{m-2}\left|\begin{array}{cccc}
\Delta_{-1} & \cdots & \Delta_{1-m} & \Delta_{-k, \tilde{z}} \\
\Delta_{0} & \cdots & \Delta_{2-m} & \Delta_{1-k, \tilde{z}} \\
\vdots & & \vdots & \vdots \\
\Delta_{m-2} & \cdots & \Delta_{0} & \Delta_{m-1-k, \tilde{z}}
\end{array}\right|\left|\begin{array}{cccccc}
0 & \cdots & 1 & \cdots & 0 & 0 \\
\Delta_{0} & \cdots & \Delta_{-k} & \cdots & \Delta_{2-m} & \Delta_{1-m} \\
\vdots & & \vdots & & \vdots & \vdots \\
\Delta_{m-2} & \cdots & \Delta_{m-2-k} & \cdots & \Delta_{0} & \Delta_{-1}
\end{array}\right| .
\end{aligned}
$$

On the other hand,

$$
\begin{aligned}
& e_{m, w}^{\prime}=\left|\begin{array}{cccc}
\Delta_{-1} & \cdots & \Delta_{1-m} & \Delta_{-m, w} \\
\Delta_{0} & \cdots & \Delta_{2-m} & \Delta_{1-m, w} \\
\vdots & & \vdots & \vdots \\
\Delta_{m-3} & \cdots & \Delta_{-1} & \Delta_{-2, w} \\
\Delta_{m-2} & \cdots & \Delta_{0} & \Delta_{-1, w}
\end{array}\right| \\
&+\sum_{k=0}^{m-2}\left|\begin{array}{cccccccc}
\Delta_{-1} & \cdots & \Delta_{1-m} & \Delta_{-k-1, w} \\
\Delta_{0} & \cdots & \Delta_{2-m} & \Delta_{-k, w} \\
\vdots & & \vdots & \vdots \\
\Delta_{m-2} & \cdots & \Delta_{0} & \Delta_{m-2-k, w}
\end{array}\right|\left|\begin{array}{cccccc}
0 & \cdots & 1 & \cdots & 0 & 0 \\
\Delta_{0} & \cdots & \Delta_{-k} & \cdots & \Delta_{2-m} & \Delta_{1-m} \\
\vdots & & \vdots & & \vdots & \vdots \\
\Delta_{m-2} & \cdots & \Delta_{m-2-k} & \cdots & \Delta_{0} & \Delta_{-1}
\end{array}\right|,
\end{aligned}
$$

and then the result follows immediately from (3.6).

4. Conclusion and discussion. In this paper, we have presented Bäcklund transformations for the NC ASDYM equation with $G=G L(2)$ and constructed from a simple seed solution a series of exact NC Atiyah-Ward ansatz solutions expressed explicitly in terms of quasi-determinants.

In this short paper we have not discussed several important points concerning the NC CFYG transformation, for example, the origin of this transformation in the framework of $\mathrm{NC}$ twistor theory, analysis of explicit exact solutions, the relationship with NC Darboux and NC binary Darboux transformation [29] and so on. These are reported in our forthcoming more detailed paper [10]. NC extension of a bilinear form approach to the ASDYM equation [31] is also an interesting topics because many aspects in that paper are close to ours.

These results could be applied also to lower-dimensional systems via the results on the NC Ward's conjecture including NC monopoles, NC KdV equations and so on, and might shed light on a profound connection between higher-dimensional integrable systems related to twistor theory and lower-dimensional ones related to Sato's theory. 
Appendix A. Proof of the compact form for the NC Atiyah-Ward ansatz solutions Consider the $2 \times 2$ matrices of quasi-determinants of the form

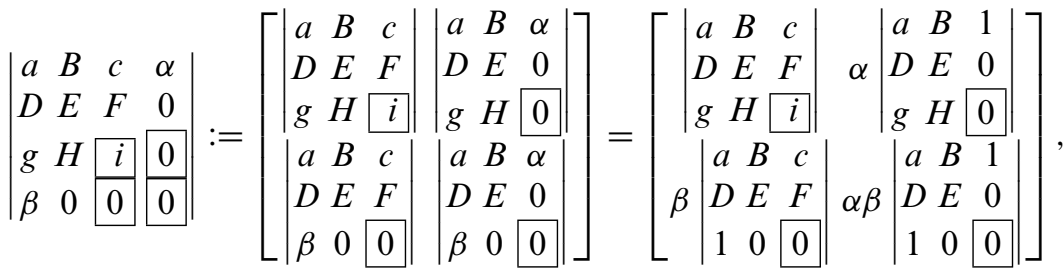

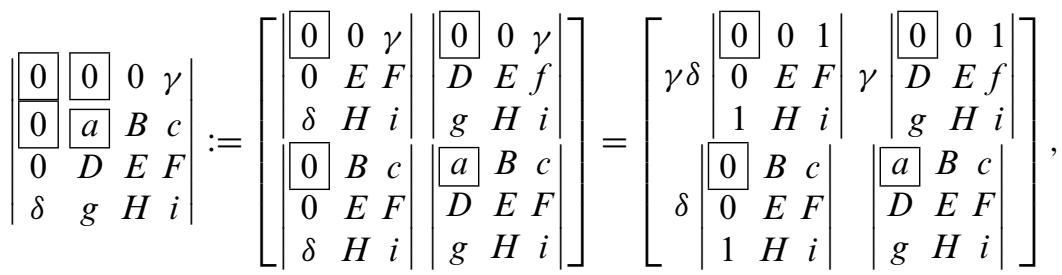

where lowercase letters denote single entries, uppercase letters denote matrices of compatible dimensions and Greek letters are scalars (i.e. commute with everything). Using (2.4) these can be rewritten as

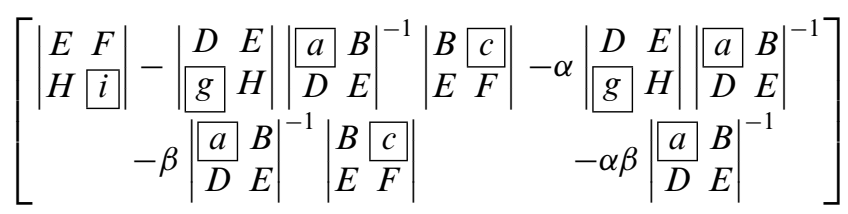

and

$$
\left[\begin{array}{c}
-\gamma \delta\left|\begin{array}{ll}
E & F \\
H & i
\end{array}\right|^{-1}-\gamma\left|\begin{array}{ll}
E & F \\
H & i
\end{array}\right|^{-1}\left|\begin{array}{ll}
D & E \\
\hline g & H
\end{array}\right| \\
\left.-\delta\left|\begin{array}{ll}
B & c \\
E & F
\end{array}\right|\left|\begin{array}{ll}
E & F \\
H & i
\end{array}\right|^{-1}\left|\begin{array}{ll}
a & B \\
D & E
\end{array}\right|-\left|\begin{array}{ll}
B & c \\
E & F
\end{array}\right|\left|\begin{array}{ll}
E & F \\
H & i
\end{array}\right| \begin{array}{ll}
-1 \\
\left|\begin{array}{ll}
g & H
\end{array}\right|
\end{array}\right]
\end{array}\right]
$$

The product of these matrices is an identity matrix $I$ if and only if $\alpha \beta=\gamma \delta=-1$ and $\alpha+\gamma=0$ and so we choose $\alpha=-\beta=-\gamma=\delta=1$. Thus

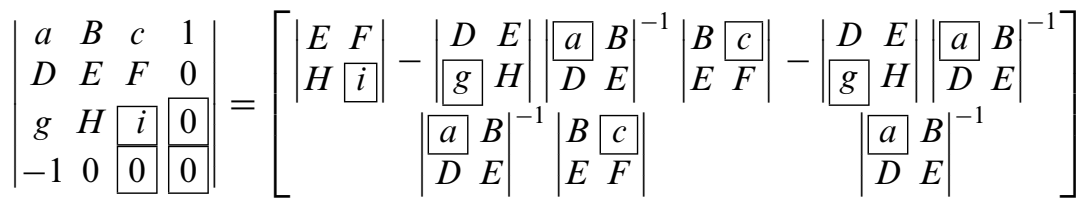

and

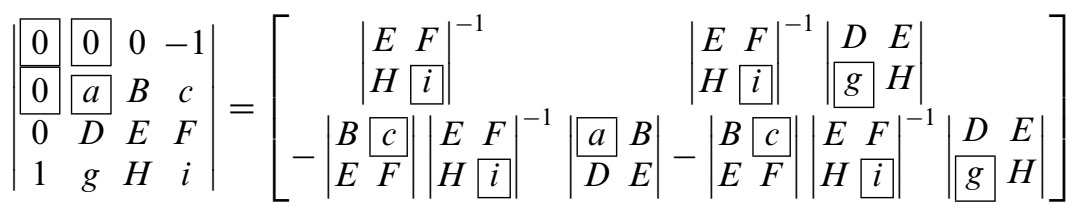

are inverse to each other. 
To apply these results to the Atiyah-Ward ansatz solutions of $R_{m}^{\prime}$, we get the representation (3.7) and (3.8).

AcKnowledgements. Masashi Hamanka would like to thank to L. Mason for a lot of helpful comments and hospitality during stay at Mathematical Institute, University of Oxford and to Jonathan J. C. Nimmo for hospitality during stay at Department of Mathematics, University of Glasgow and to organizers for hospitality during the ISLAND3 conference in Islay. The work of Masashi Hamanaka was supported by the Yamada Science Foundation for the promotion of the natural science and Grant-in-Aid for Young Scientists (\#18740142).

\section{REFERENCES}

1. M. F. Atiyah and R. S. Ward, Instantons and Algebraic Geometry, Commun. Math. Phys. 55 (1977), 117.

2. S. J. Brain, The noncommutative Penrose-Ward transform and self-dual Yang-Mills fields, $\mathrm{PhD}$ Thesis (University of Oxford, 2005).

3. S. J. Brain and S. Majid, Quantisation of twistor theory by cocycle twist, Commun. Math. Phys. 284 (2008), 713.

4. E. Corrigan and D. B. Fairlie, Scalar field theory and exact solutions to a classical SU(2) gauge theory, Phys. Lett. B 67 (1977), 69; G. 't Hooft, unpublished; F. Wilczek, in Quark Confinement and Field Theory (Wiley, Princeton, 1977) 211 [ISBN/0471-02721-9].

5. E. Corrigan, D. B. Fairlie, R. G. Yates and P. Goddard, Backlund Transformations and the Construction of the Atiyah-Ward Ansatz for Selfdual SU(2) Gauge Fields, Phys. Lett. B 72 (1978), 354; Commun. Math. Phys. 58 (1978), 223.

6. A. Dimakis and F. Müller-Hoissen, From nonassociativity to solutions of the KP hierarchy, J. Phys. A 40 (2007), F321.

7. P. Etingof, I. Gelfand and V. Retakh, Factorization of differential operators, quasideterminants, and nonabelian Toda field equations, Math. Res. Lett. 4 (1997), 413; Math. Res. Lett. 5 (1998), 1.

8. I. Gelfand, S. Gelfand, V. Retakh and R. L. Wilson, Quasideterminants, Adv. Math. 193 (2005), 56.

9. I. Gelfand and V. Retakh, Determinants of matrices over noncommutative rings, Funct. Anal. Appl. 25 (1991), 91; Funct. Anal. Appl. 26 (1992), 231.

10. C. R. Gilson, M. Hamanaka and J. J. C. Nimmo, Backlund transformations and the Atiyah-Ward Ansatz for noncommutative anti-self-dual Yang-Mills equations, arXiv:0812.1222.

11. C. R. Gilson and J. J. C. Nimmo, On a direct approach to quasideterminant solutions of a noncommutative KP equation, J. Phys. A 40 (2007), 3839.

12. C. R. Gilson, J. J. C. Nimmo and Y. Ohta, Quasideterminant solutions of a non-Abelian Hirota-Miwa equation, J. Phys. A $\mathbf{4 0}$ (2007), 12607.

13. C. R. Gilson, J. J. C. Nimmo and C. M. Sooman, On a direct approach to quasideterminant solutions of a noncommutative modified KP equation, J. Phys. A 41 (2008), 085202.

14. V. M. Goncharenko and A. P. Veselov, Monodromy of the matrix Schrodinger equations and Darboux transformations, J. Phys. A 31 (1998), 5315.

15. M. Hamanaka, Noncommutative Ward's conjecture and integrable systems, Nucl. Phys. B 741 (2006), 368.

16. M. Hamanaka, Notes on exact multi-soliton solutions of noncommutative integrable hierarchies, JHEP 0702 (2007), 094.

17. K. C. Hannabuss, Non-commutative twistor space, Lett. Math. Phys. 58 (2001), 153.

18. Z. Horváth, O. Lechtenfeld and $M$. Wolf, Noncommutative instantons via dressing and splitting approaches, JHEP 0212 (2002), 060. 
19. M. Ihl and S. Uhlmann, Noncommutative extended waves and soliton-like configurations in $\mathrm{N}=2$ string theory, Int. J. Mod. Phys. A 18 (2003), 4889.

20. A. Kapustin, A. Kuznetsov and D. Orlov, Noncommutative instantons and twistor transform, Commun. Math. Phys. 221 (2001), 385.

21. A. Konechny and A. S. Schwarz, Introduction to M(atrix) theory and noncommutative geometry, Phys. Rept. 360 (2002), 353; J. A. Harvey, Komaba lectures on noncommutative solitons and D-branes, hep-th/0102076; M. R. Douglas and N. A. Nekrasov, Noncommutative field theory, Rev. Mod. Phys. 73 (2002), 977; R. J. Szabo, Quantum field theory on noncommutative spaces, Phys. Rept. 378 (2003), 207; M. Hamanaka, Noncommutative solitons and D-branes, PhD Thesis, hep-th/0303256. C. S. Chu, Non-commutative geometry from strings, hep-th/0502167; R. J. Szabo, D-Branes in noncommutative field theory, hep-th/0512054.

22. B. Kupershmidt, $K P$ or $m K P$ : noncommutative mathematics of Lagrangian, Hamiltonian, and integrable systems (AMS, 2000) [ISBN/0821814001]; M. Hamanaka, Noncommutative solitons and integrable systems, [hep-th/0504001]; L. Tamassia, Noncommutative supersymmetric/integrable models and string theory, PhD Thesis, hepth/0506064; O. Lechtenfeld, Noncommutative solitons, hep-th/0605034; A. Dimakis and F. Müller-Hoissen, From nonassociativity to solutions of the KP hierarchy, nlin.SI/0608017.

23. O. Lechtenfeld and A. D. Popov, Noncommutative 't Hooft instantons, JHEP 0203 (2002), 040.

24. O. Lechtenfeld, A. D. Popov and B. Spendig, Open $\mathrm{N}=2$ strings in a B-field background and noncommutative self-dual Yang-Mills, Phys. Lett. B 507 (2001), 317; JHEP 0106 (2001), 011.

25. C. X. Li and J. J. C. Nimmo, Quasideterminant solutions of a non-Abelian Toda lattice and kink solutions of a matrix sine-Gordon equation, Proc. Roy. Soc. Lond. A 464 (2008), 951.

26. L. J. Mason and N. M. Woodhouse, Integrability, self-duality, and twistor theory (Oxford University Press, New York, 1996) (London Mathematical Society monographs, new series: 15), [ISBN/0-19-853498-1].

27. N. Nekrasov and A. Schwarz, Instantons on noncommutative $\mathrm{R}^{* * 4}$ and $(2,0)$ superconformal six dimensional theory, Commun. Math. Phys. 198 (1998), 689.

28. J. J. C. Nimmo, On a non-Aberian Hirota-Miwa equation, J. Phys. A 39 (2006), 5053.

29. U. Saleem, M. Hassan and M. Siddiq, Non-local continuity equations and binary Darboux transformation of noncommutative (anti) self-dual Yang-Mills equations, J. Phys. A 40 (2007), 5205.

30. B. F. Samsonov and A. A. Pecheritsin, Chains of Darboux transformations for the matrix Schrödinger equation, J. Phys. A 37 (2004), 239.

31. N. Sasa, Y. Ohta and J. Matsukidaira, Bilinear Form Approach to the Self-Dual YangMills Equation and Integrable System in (2+1)-Dimension, J. Phys. Soc. Jap. 67 (1998), 83.

32. K. Takasaki, Anti-self-dual Yang-Mills equations on noncommutative spacetime, J. Geom. Phys. 37 (2001), 291.

33. R. S. Ward, Integrable and Solvable Systems, and Relations Among Them, Phil. Trans. Roy. Soc. Lond. A 315 (1985), 451.

34. C. N. Yang, Condition of Selfduality For SU(2) Gauge Fields on Euclidean FourDimensional Space, Phys. Rev. Lett. 38 (1977), 1377. 